\title{
An Efficient Diversity Exploitation in Multiuser Time-Varying Frequency-Selective Fading Channels
}

\author{
Wenxun Qiu, Student Member, IEEE, Hlaing Minn, Senior Member, IEEE, \\ and Chia-Chin Chong, Senior Member, IEEE
}

\begin{abstract}
Diversity exploitation is a proven efficient means for addressing detrimental effects of wireless channel fadings. User channelization, where the frequency and time resources are structured into several user channels, can substantially help to capture the diversity, but it has not been well investigated. In this paper, we develop theoretical formulation of optimal resource structures under different performance metrics and then analyze them in frequency-selective time-varying channels. We propose a simplified system model which maintains the main features, and based on which, we develop a new practical approach of user channelization or user resource structuring which captures better diversity for OFDMA systems in multiuser time-varying frequency-selective fading channels. In contrast to the existing user resource structures where relative locations of the resources in the time-frequency grid remain the same throughout the transmission frame, the proposed resource structures evolve within the frame to enjoy better diversity gain. Example designs of the proposed evolving structures are presented from the 3GPP LTE perspective. Simulations done under LTE system environments validate that the proposed practical approach can obtain a near-optimal structure design, and provide significant performance advantage over the existing structures at the mobile speeds ranging from $3 \mathrm{~km} / \mathrm{h}$ to $350 \mathrm{~km} / \mathrm{h}$.
\end{abstract}

Index Terms-Channelization, diversity, OFDMA, resource structure, time-varying channel.

\section{INTRODUCTION}

$\mathbf{S}$ UPPORTING larger data rates at higher mobile speeds has been among the main technical challenges of every new generation mobile communication systems. The IMTAdvanced (a.k.a. 4G) systems plan to support $1 \mathrm{Gbps}$ at low mobile speeds and $100 \mathrm{Mbps}$ at high mobile speeds [1] as compared to $2 \mathrm{Mbps}$ or $348 \mathrm{kbps}$ for the IMT-2000 (3G) requirements. The mobile speed range of $4 \mathrm{G}$ is up to $350 \mathrm{~km} / \mathrm{h}$, which is much higher than that of $3 \mathrm{G}$ counterpart. The challenge is much more significant when the applications impose a stringent delay constraint. Efficient diversity exploitation is crucial in addressing this challenge. Depending on the transmission scheme, diversity capturing mechanism could vary. As orthogonal frequency division multiple access (OFDMA)

Paper approved by J. N. Laneman, the Editor for Cooperative Relaying and Diversity Techniques of the IEEE Communications Society. Manuscript received July 31, 2009; revised August 5, 2010 and November 22, 2010.

Parts of the material in this paper were published in the Proceedings, IEEE WCNC, 2009 [28].

W. Qiu and H. Minn are with the Department of Electrical Engineering, University of Texas at Dallas, Richardson, TX, 75080 USA (e-mail: \{wxq081000, hlaing.minn\}@utdallas.edu).

C.-C. Chong was with DOCOMO USA Labs, 3240 Hillview Avenue, Palo Alto, CA, 94304 USA (e-mail: chia-chin.chong@ieee.org).

Digital Object Identifier 10.1109/TCOMM.2011.060911.090439 is adopted in several next-generation standards, e.g., 3GPP Long Term Evolution (LTE) [2], 3GPP LTE-Advanced [3], Mobile WiMAX [4], this paper focuses on efficient diversity exploitation in multiuser time-varying OFDMA systems.

There exist several forms of diversity to exploit and this paper deals with frequency, multiuser, and time diversities. The existing literature on frequency diversity exploitation in OFDMA includes bit-interleaved coded modulation (BICM) [5], precoding with subcarrier selection [6], block spreading [7], and subcarriers and bit allocation [8], [9]. The multiuser diversity exploitation is addressed by resource allocation (e.g., [10]-[15]) or scheduling (e.g., [16], [17]) according to the channel state information (CSI) of the users. While exploiting frequency diversity may not require CSI, exploiting multiuser diversity does require CSI knowledge. In the latter case, typically each user estimates CSI and feedback to the base station (BS). For frequency-selective channels, the corresponding overhead for full CSI feedback may not be affordable, and hence several limited feedback schemes (e.g., [18]-[21]) have been proposed. However, in fast time-varying channels, outdated CSI knowledge would degrade the diversity gains [22][23], and in particular, if the CSI feedback rate is much slower than necessary, the subcarriers assigned to a user may all be in deep fade at the later part of the transmission frame. On the other hand, more frequent CSI updates come with the cost of increased overhead and energy, which may not be practical for the advanced cellular systems. This leads to a tradeoff between the CSI-based diversity and mobility [24].

User channelization (or user resource structure in the frequency and time domain) does affect the diversity exploitability, but using it as a tool to enhance diversity in multiuser time-varying frequency-selective fading channels has not been considered in the literature. Here, user channelization (user resource structure) should not be confused with resource allocation or scheduling. Scheduling decides which users are allowed to transmit, while resource allocation assigns resource to the scheduled users. Both resource allocation and scheduling can be performed on a per-tone basis, but the required signaling overhead to inform users of which tones are allocated to what users is too large for the per-tone approach. In addition, the effects of inter-carrier interference (ICI) (e.g., due to carrier frequency offset) among different users can be more detrimental in the per-tone approach. Furthermore, channel gains are highly correlated over adjacent tones. Hence, in practice, resource allocation is more efficiently done based on larger resource block than single tones. User channelization 
corresponds to how to construct these resource blocks (user resource structures or sub-channels).

The existing diversity exploitation techniques try to capture diversities constrained under the existing user channelization schemes. There are mainly two types of user resource structures, namely the interleaved-type (a.k.a distributed type in LTE) and the band-type (a.k.a. localized type in LTE). The subcarrier locations of a user channel ${ }^{1}$ in the first type are spread out over the entire OFDMA band, hence interleaving with those of other user channels. Those in the second type are adjacently located, forming a band. ${ }^{2}$ The interleaved-type resource structure provides frequency diversity and robustness against deep fades but cannot capture significant gain brought in by the multiuser diversity. The band-type resource structure gives significant multiuser diversity gain but it may be severely affected by deep fades at later part of the transmission frame in fast time-varying channels. The main source for the limitations of the existing resource structures is that they are each designed to exploit only one type of diversity and the channel time-variations within the transmission frame are not taken into consideration.

The contributions of this paper are as follows:

1) New concept for technical advancement

We propose a new paradigm namely user resource structure design for diversity enhancement. It reveals the limitations of the existing resource structures, enhances the capturable diversity of a user channel, and releases an additional dimension for system optimization.

2) New theoretical development

a) We introduce theoretical formulations of user channelization using bit error rate (BER) of repetition code and outage probability, which unveil main characteristics of optimal resource structures.

b) According to the main characteristics of optimal resource structures, we propose a simplified system model for designing user resource structures.

c) We present a BER performance analysis under the simplified system model, which leads to the practical resource structure design approach.

3) New practical design procedures/algorithms

Based on the analysis under the simplified system model, we propose a practical resource structure design procedure and also a simplified version of the resource structure design, which is more practical. The simulation illustrates that the proposed resource structure design and its simplified version can both improve the SNR performance significantly in LTE system environment and obtain a close-to-optimal performance. Additionally, it is validated that the proposed resource structures are not so sensitive to the mismatches in diversity order and mobile speed.

The paper is organized as follows. The system and channel models are introduced in Section II. The theoretical framework

\footnotetext{
${ }^{1} \mathrm{~A}$ user channel here represents a minimum resource to be allocated to a user. More than one user channels may be allocated to a particular user depending on its data rate requirement.

${ }^{2}$ In a more general setup, the subcarrier locations of a user channel may be shifted from symbol to symbol in a systematic way but their relative locations in each OFDM symbol follow the same principle of interleaved or band type.
}

of proposed approach for diversity enhancement is presented in Section III, which includes the rationale and theoretical formulation of the user channelization and BER performance in a proposed simplified system model. The practical resource structure design and its simplified design are proposed in Section IV. In Section V, simulations validate the advantage of the proposed resource structure over the existing structures. Finally, conclusions are provided in Section VI.

\section{SyStem Model}

We consider a multiuser OFDMA system in a timevarying frequency-selective fading channel. We assume that CSI knowledge of all users is available at the BS but it is outdated by $\mu$ OFDM symbol delay. CSI could be obtained in several ways. In frequency division duplexing (FDD) systems, an explicit channel can be used to feedback the channel estimates from the users. In time division duplexing (TDD) systems, the BS can estimate CSI directly from the received uplink (UL) signals or obtain CSI from the feedback of the users. On the other hand, at the receiver side we assume the CSI knowledge is perfect ${ }^{3}$ (could be obtained from downlink (DL) pilots).

\section{A. Channel Model}

As next-generation systems aim to offer reliable services in a wide range of mobile speeds, the channel gain may vary substantially within the transmission frame ${ }^{4}$. We assume that the channel gains remain static within one OFDM symbol, but may vary from one symbol to another. This assumption is reasonable, since even at $350 \mathrm{~km} / \mathrm{h}$ in LTE environments, the OFDM symbol duration is still much less than the coherence time. Let $H[i, n]$ denote the low-pass equivalent channel gain on the $i$ th subcarrier of the $n$th OFDM symbol. With welldefined joint probability density function (pdf) of $\{H[i, n]\}$, there is no limitation on the physical channel model for the proposed approach to be applicable. In our theoretical formulation of the user channelization, the joint pdf of $\{H[i, n]\}$ is required to solve the design problem numerically, or generation of $\{H[i, n]\}$ is needed to solve it by simulation. In our practical design, only channel correlation properties are required.

For theoretical performance analysis and simulation, we adopt a frequency-selective Rayleigh fading channel, for which $\{H[i, n]\}$ are jointly Gaussian with $E\left[|\Re\{H[i, n]\}|^{2}\right]=$ $E\left[|\Im\{H[i, n]\}|^{2}\right]=\sigma_{h}^{2}, E[\Re\{H[i, n]\} \Im\{H[l, m]\}]=0$, and $E[\Re\{H[i, m]\} \Re\{H[i, m+n]\}]=E[\Im\{H[i, l]\} \Im\{H[i, l+n]\}]$ $=\rho_{i}[n] \sigma_{h}^{2}$, for all $i, l, n, m$. As we assume that the CSI knowledge at the BS is outdated by $\mu$ symbols at the beginning of the transmission, $H[i,-\mu]$ can be considered as the CSI knowledge of the $i$ th subcarrier at the BS. The temporal correlation coefficient of the $i$ th subcarrier channel gain between the $n$th OFDM symbol and the corresponding CSI knowledge is then given by $\rho_{i}[n+\mu]$. A common model of the channel temporal correlation is given by the classical

\footnotetext{
${ }^{3}$ Our design is not limited to the system where CSI is perfectly known to the receiver.

${ }^{4}$ In LTE, a frame consists of both uplink and downlink sub-frames, but in this paper a frame corresponds to either uplink or downlink subframe.
} 


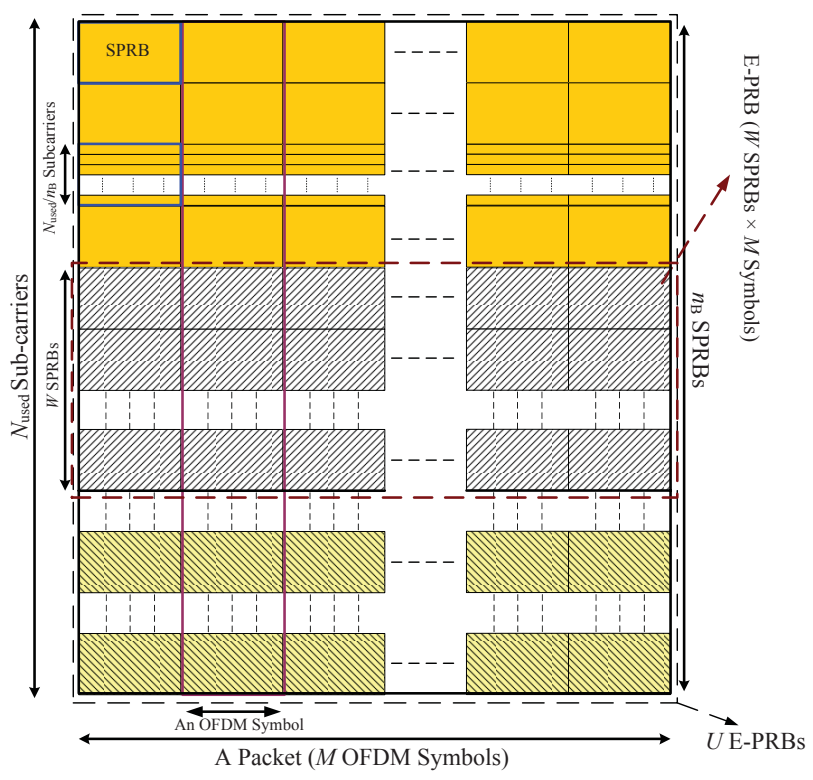

Fig. 1. Transmission packet structure (band-type).

Clark-Jakes' model, which will be used in our theoretical analysis and simulation. In the model, the channel coefficients are samples of a stationary Gaussian process with a normalized autocorrelation function given by $J_{0}\left(2 \pi f_{m} \tau\right)$, where $J_{0}(x)$ is the zeroth-order Bessel function of the first kind, $\tau$ denotes the time lag, and $f_{m}$ is the maximum Doppler frequency. Note that in our practical design, $\rho_{i}[n]$ can assume other model as well.

For the considered Rayleigh fading channel, $H[i, n]$ can be linked to the CSI knowledge at the BS, $H[i,-\mu]$, as

$$
H[i, n]=\rho_{i}[n+\mu] H[i,-\mu]+w[i, n],
$$

where $w[i, n]$ has a pdf of $\mathcal{C N}\left(0,\left(1-\rho_{i}^{2}[n+\mu]\right) 2 \sigma_{h}^{2}\right)$ and is independent of $H[i,-\mu]$. Note that $w[i, m]$ and $w[i, n]$ are correlated to maintain the correlation between $H[i, m]$ and $H[i, n]$. Given the CSI knowledge $H[i,-\mu]$, the relationship in (1) facilitates an easy computation of the pdf of the channel power gain at the $n$th symbol, which will be used in the Section III-B and III-C.

\section{B. OFDMA Resource Blocks and User Channelization}

As mentioned in Section I, designing user resource structure (a.k.a. resource block (RB), user channel, or sub-channel) or user channelization should not be confused with resource allocation or scheduling. In practice, certain user resource structure or channelization is constructed and resource allocation or scheduling is performed on the basis of the user resource structure or sub-channel. A user may be allocated with more than one sub-channels. This paper only concerns with the design of user resource structure or channelization. The existing resource allocation or scheduling algorithms can be applied on top of the proposed resource structure.

The OFDMA system has $U$ RBs or subchannels with $M$ OFDM symbols per transmission frame. An example of the packet structure with the band-type resource structure is shown in Fig. 1 in order to illustrate several parameters used. Here, we follow the terminology as used in LTE in which when referring an RB physically (analogous to a physical sub-channel) we call physical resource block (PRB). However, because the size and structure of our proposed evolving structure are different from those in LTE, we will use evolving PRB (E-PRB) for the proposed structure. The discrete Fourier transform (DFT) size is $N$, and the number of usable subcarriers is $N_{\text {used }}$ per OFDM symbol. Theoretically, all tones can be used, but practical systems insert null guard tones for easier filter implementation and spectral control. To be general, we consider both scenarios of with and without null guard tones ${ }^{5}$. Then, for both systems with or without null guard tones, we can have the number of subcarriers per subchannel $N_{\mathrm{SC}}=N_{\text {used }} / U$.

For convenience of presenting our resource structure, we introduce sub physical resource block (SPRB). Each PRB is divided into several SPRBs in both time and frequency domain. There are $n_{\mathrm{B}}$ SPRBs per OFDM symbol. Each SPRB spans over one OFDM symbol only, while each PRB (or EPRB) spans over $M$ symbols. There are $N_{\text {used }} / n_{\mathrm{B}}$ consecutive subcarriers per SPRB in one symbol, and $W=n_{\mathrm{B}} / U$ SPRBs per symbol of each PRB (or E-PRB), i.e., $M W$ SPRBs per PRB. Note that each SPRB should contain at least a few consecutive subcarriers rather than a single subcarrier to achieve robustness against ICI. This also reduces the dimensionality of the optimization space for the user channelization.

Let $\boldsymbol{J}_{k}$ represent an $M \times W$ matrix defining the $k$ th subchannel where the $n$th row denoted by $\boldsymbol{J}_{k}(n,:)$ contains the SPRB indexes for the $n$th OFDM symbol of the $k$ th subchannel. The user channelization or resource structure design is equivalent to determining $\left\{\boldsymbol{J}_{k}: k=1, \ldots, U\right\}$ where $\boldsymbol{J}_{k}(n,:)$ and $\boldsymbol{J}_{m}(n,:)$ are disjoint for all $n$ and all $k \neq m$. The existing band-type channelization is given by

$$
\begin{array}{r}
\boldsymbol{J}_{k}(n,:)=[(k-1) W,(k-1) W+1, \ldots, k W-1], \\
n=0,1, \ldots, M-1,
\end{array}
$$

and the interleaved-type is defined by

$$
\begin{aligned}
\boldsymbol{J}_{k}(n,:)= & {[(k-1), k-1+U, k-1+2 U, \ldots, k-1} \\
& +(W-1) U], n=0,1, \ldots, M-1 .
\end{aligned}
$$

In the above conventional structures, the subcarrier locations of each PRB are fixed. In a more general setup, they can be all shifted in a certain manner from symbol to symbol. In any case, the relative positions of subcarriers of each PRB remain the same across the symbols of a frame in these structures.

\section{Theoretical Framework of Proposed APPROACH}

\section{A. Rationale and Overview of Proposed Approach}

The main feature of the proposed approach is the shaping of user resource structure to capture better diversities in multiuser time-varying frequency-selective fading channels. The existing resource structures focus on exploiting only one form of diversity and do not consider the channel time-variations

\footnotetext{
${ }^{5}$ We simply use null guard tones to represent any reserved tones at the band edges.
} 
within the transmission frame. As a result, the relative locations of their resources within a user channel remain static throughout the frame, limiting their ability to capture other available diversities. By exploiting CSI knowledge of all users in the resource allocation at the $\mathrm{BS}$, multiuser diversity can be captured via the band-type resource structure. When the CSI knowledge becomes outdated (the actual channel gains become uncorrelated with the CSI known to the BS) at a later part of the frame, all subcarriers of the band-type structure are susceptible to deep fading. On the other hand, the interleavedtype provides robustness to frequency-selective deep fades but loses significant multiuser diversity gain. Note that in the band-type case, the diversity gain comes from both multiuser and channel frequency-selectivity with CSI knowledge, but it will be referred to as multiuser diversity in order to distinguish it from the frequency diversity achieved with the interleavedtype when there is no CSI knowledge. In order to overcome the limitations of the existing fixed resource structures, we propose the concept of an evolving resource structure in order to capture best diversity gains in multiuser time-varying frequency-selective fading channels.

In the rest of this section, we first develop theoretical formulation of user resource structure design and study the main characteristics of optimal resource structures. According to these characteristics, we propose a simplified system model and derive the BER performance of repetition code on this model as the metric for the resource structure design.

\section{B. Theoretical Formulation of User Channelization}

As a subchannel is a minimum resource (for data channel) to be allocated to a user, it is logical to assume that the bandwidth of a subchannel, if all of its subcarriers are consecutively located, is around the channel coherence bandwidth. A much smaller subchannel bandwidth will require more signaling overhead in resource allocation and will be more susceptible to ICI, while a larger bandwidth will sacrifice CSI-based diversity gain. We assume that CSI knowledge is available at the beginning of the frame. As theoretical formulations of the user channelization, we adopt BER of a repetition code and outage probability at an average signal-to-noise ratio (SNR) with the rate $R_{T}$ as our metrics. To illustrate the captured diversity order, we adopt the repetition code with code rate of $1 / W$. Via designs under both metrics, main characteristics of the optimal resource structure can be revealed.

The channelization should equally divides the bandwidth into $U$ channels. Instead of designing for $U$ users at the same time, we consider the case of only one user occupying $1 / U$ of the whole bandwidth (designing $\boldsymbol{J}_{1}$ ). Additionally, we also introduce the constraint that the whole bandwidth can be exactly covered by $\left\{\boldsymbol{J}_{k}, k=1, \cdots, U\right\}$, so that we can obtain the rest $U-1$ channels by shifting or flipping and shifting $\boldsymbol{J}_{1}$. Then we can formulate the optimal user channelization as

$$
\begin{aligned}
& \boldsymbol{J}_{1}(n,:)=\arg \min _{\overline{\boldsymbol{J}}} E\left[\boldsymbol{\mathcal { M }}\left(n, \overline{\boldsymbol{J}}+\tilde{I}_{0}\right) \mid\{H[i,-\mu]\}\right] \\
& n=0, \ldots, M-1, \\
\text { s.t. } & \boldsymbol{J}_{k}(n,:)=\hat{\boldsymbol{J}}_{1}(n,:)+D_{k}(n), k=1, \ldots, U, \\
& \boldsymbol{J}_{k}(n,:) \cap \boldsymbol{J}_{m}(n,:)=\emptyset, \forall k \neq m, n=0, \ldots, M-1,
\end{aligned}
$$

where $\overline{\boldsymbol{J}}$ represents any $W$ combination from $\left\{0,1, \ldots, n_{\mathrm{B}}-\right.$ $1\}, \hat{\boldsymbol{J}}_{1}(n,:)$ is either $\boldsymbol{J}_{1}(n,:)$ or its laterally-flipped (mirrored) version about its edge, and the expectation $E[\cdot]$ in (4) is over $\{H[i,-\mu]\}$. Here, $\mathcal{M}\left(n, \overline{\boldsymbol{J}}+\tilde{I}_{0}\right)$ is the selected metric, such as BER of repetition code or outage probability at symbol $n$ for the subchannel with the SPRB indexes defined by $\overline{\boldsymbol{J}}+\tilde{I}_{0}$. And $\tilde{I}_{0}$ is the first index of $\tilde{I}$ which is defined as

$$
\tilde{I} \triangleq \arg \min _{\overline{\boldsymbol{J}}}[\boldsymbol{\mathcal { M }}(0, \overline{\boldsymbol{J}}) \mid\{H[i,-\mu]\}] .
$$

Note that the addition of $\tilde{I}_{0}$ in (4) is just to make the SPRB indexes for the other OFDM symbols to be with reference to those for the first symbol. The reason is that due to the diversity exploitation with the CSI knowledge, $\tilde{I}$ will vary from one channel realization to another.

The constraints in (5) and (6) are set to fit the SPRB indexes of $U$ subchannels within the available SPRB indexes for each symbol without any overlapping and to have all subchannels with the same or mirrored shape within the transmission frame. $\left\{D_{k}(n)\right\}$ denote the shifts at each symbol for other subchannels with respect to the first subchannel. The inclusion of the mirrored version improves flexibility in fitting SPRBs of all subchannels disjointly while optimizing the performance metric. All the SPRB indexes throughout this paper are implicitly assumed to be modulo $n_{\mathrm{B}}$.

If $\mathrm{BER}$ of repetition code with $1 / W$ code rate and binary phase shift keying (BPSK) is adopted, we have

$$
\begin{gathered}
\mathcal{M}(n, \overline{\boldsymbol{J}} \mid\{H[i,-\mu]\}) \\
=E\left[Q\left(\sqrt{\sum_{i \in \overline{\boldsymbol{J}}}|H[i, n]|^{2} \mathrm{SNR}}\right) \mid\{H[i,-\mu]\}\right], \\
\tilde{I} \triangleq \arg \min _{\overline{\boldsymbol{J}}} E\left[Q\left(\sqrt{\sum_{i \in \overline{\boldsymbol{J}}}|H[i, 0]|^{2} \mathrm{SNR}}\right) \mid\{H[i,-\mu]\}\right] .
\end{gathered}
$$

Here, the expectation $E[\cdot]$ in (8) is over $\{H[i, n]\}$ and in (9) is over $\{H[i, 0]\}$, both conditioned on $\{H[i,-\mu]\}, i \in \overline{\boldsymbol{J}}$. Similarly, by the criterion of outage probability, the metric can be formulated as

$$
\begin{aligned}
& \mathcal{M}(n, \overline{\boldsymbol{J}} \mid\{H[i,-\mu]\}) \\
& =P\left[\sum_{i \in \overline{\boldsymbol{J}}} \log _{2}\left(1+|H[i, n]|^{2} \mathrm{SNR}\right)<R_{T} \mid\{H[i,-\mu]\}\right] .
\end{aligned}
$$

In this case, $\tilde{I}$ in (7) is defined as (11). By substituting (10) and (11) into (4), we can obtain the user channelization based on the outage probability.

Neither the user resource structure designs based on metric in (8) nor (10) lend a theoretical closed-form solution. But, numerical or simulation based solution is feasible for OFDMA systems with a small number of SPRBs. Fig. 2 shows the optimal resource structures obtained from a simulation based approach to (8) and (10) for OFDMA with $N=n_{\mathrm{B}}=16$, $N_{\mathrm{SC}}=1, W=4$, and $M=14$, in a 4-tap Rayleigh fading channel at the speed of $120 \mathrm{~km} / \mathrm{h}$ and $350 \mathrm{~km} / \mathrm{h}$. Here, we take 


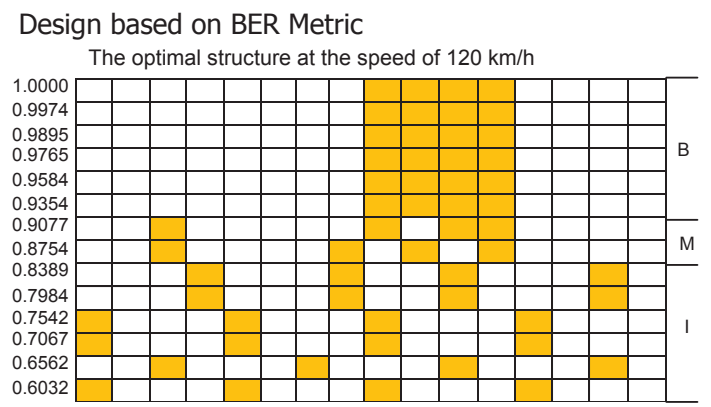

The optimal structure at the speed of $350 \mathrm{~km} / \mathrm{h}$

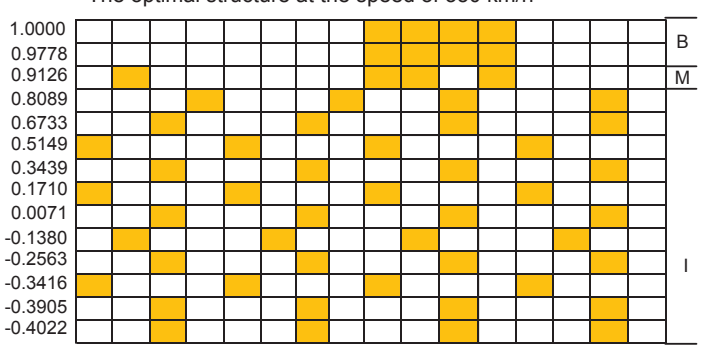

Design based on Outage Probability Metric

The optimal structure at the speed of $120 \mathrm{~km} / \mathrm{h}$

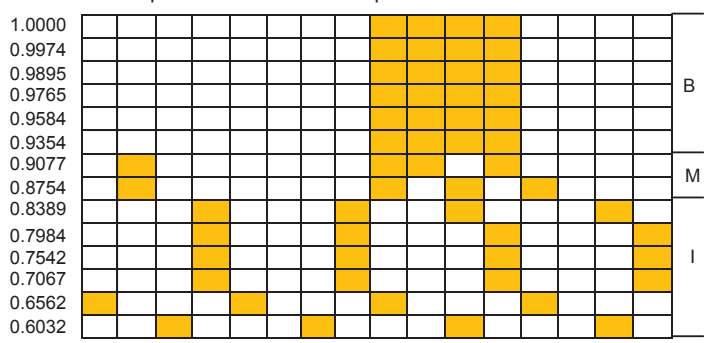

The optimal structure at the speed of $350 \mathrm{~km} / \mathrm{h}$

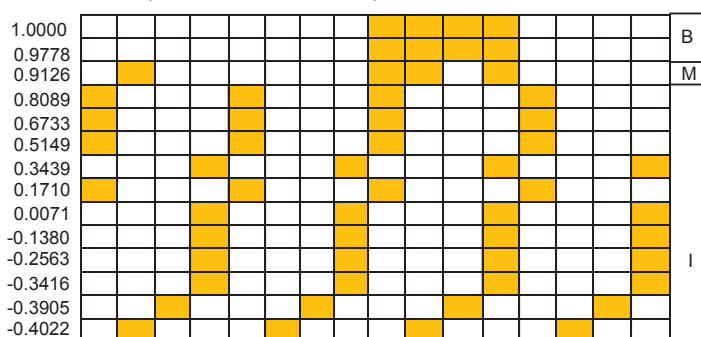

B - Band-type, M - Mixed-type, I - Interleaved-type, X axis is SPRB index, Y axis is channel temporal correlation coefficient

Fig. 2. Optimal resource structures.

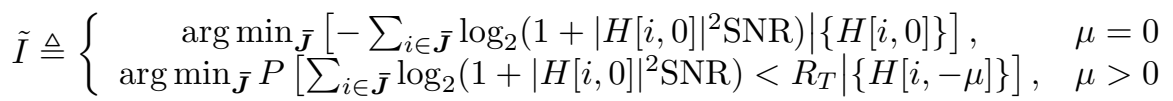

$\mu=0$ as an example and set $\mathrm{SNR}=3 \mathrm{~dB}, R_{T}=2$, symbol duration $T_{s}=71.36 \mu s$, which is adopted by LTE [2]. The channel temporal correlation coefficient (for a time interval $\tau)$ is given by $J_{0}\left(2 \pi f_{m} \tau\right)$. The Y-axis in Fig. 2 represents the channel temporal correlation coefficient for time intervals of integral OFDM symbols in an increasing order. Since the OFDM channel power gain is cyclic in frequency domain after DFT processing, we shift the structure at every realization to the third subchannel so that we can observe the structure clearly. We observe that the structure in the first symbol is of band-type. However, there are numerous ways to have $U$ band-type sub-channels (since the first subchannel can start at any subcarrier index) and this imposes unnecessarily large control overhead to inform users of the sub-channel format. A practical solution is to use a fixed band-type sub-channel (i.e., the first subchannel starts at a fixed subcarrier index) in the first symbol (hence overcoming the overhead issue), and fortunately based on our simulation (due to space limitation, the figure is not included), incorporating this additional constraint into (4) with (8) and (10) does not change the result.

Fig. 2 shows that the optimal structures coming from different criteria are quite similar. The structures can be roughly divided into three phases: Band-type at the beginning, transition phase in the middle and finally interleaved-type. Especially, in the second phase, some selected SPRBs still keep as band-type and the other SPRBs move away from the original subchannel, which can be taken as independent from the original subchannel (this type is named mixed-type). This gives us the idea about the pattern of the second phase. In the last phase, since different interleaved-type structures have almost the same statistics, we can uniformly apply one of them to all symbols. Another important feature is that the durations of each phase according to different metrics are very similar, which means we can adopt one of them to design a practical resource structure, e.g., BER of repetition code. The comparisons of different structures developed under BER and outage probability metrics at the speed of $120 \mathrm{~km} / \mathrm{h}$ are shown in Figs. 3 and 4, respectively. The above-mentioned features of three phases and the durations are validated again. Furthermore, to test the sensitivity of the structure design on the diversity order mismatch, we also evaluate the optimal designs for 3-tap and 5-tap channels in this 4-tap channel. The results in Figs. 3 and 4 show that these two designs with small diversity order mismatch still perform much better than band-type and interleave-type structure, and give a similar performance to the optimal design for 4-tap channel. This reveals another characteristic that the structure design is not so sensitive to the number of channel taps $L$ when $L$ fluctuates in a small range.

The above theoretical formulation has following limitations:

- no closed-form solution

- numerical or simulation based solution can be obtained only for systems with small dimensionality (small $n_{\mathrm{B}}$ and $W$ ) due to high computational complexity.

However, its solution under down-scaled systems reveals important characteristics of optimal user resource structure:

- The user resource structure is evolving within the frame for moderate and fast time-varying channels

- It can contain a maximum of three phases sequentially: multiuser diversity exploitation phase using the band-type structure, transition from multiuser to frequency diversity 


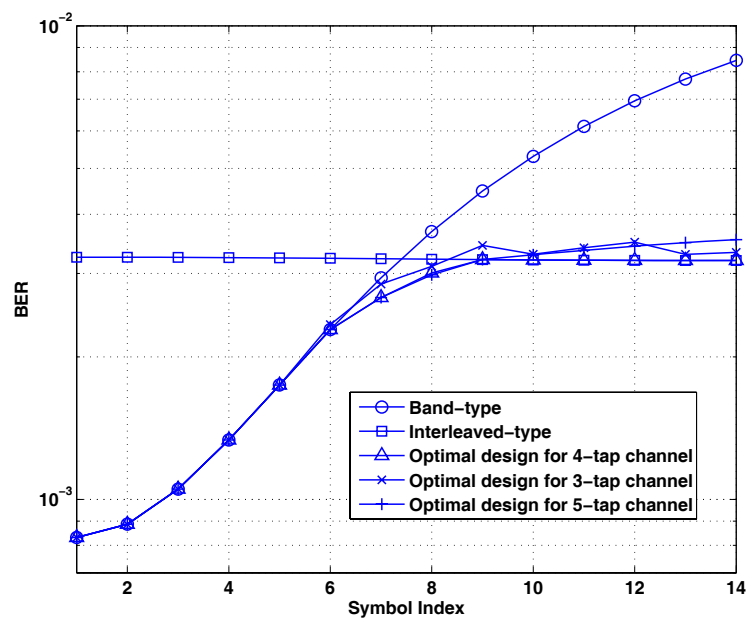

Fig. 3. BER comparison of repetition code in a 4-tap channel with SNR = $3 \mathrm{~dB}$ and $T_{\mathrm{S}}=71.36 \mu \mathrm{s}$.

exploitation using mixed-type structures, and frequency diversity exploitation phase using the interleaved-type

- The durations of the above phases depend on the channel correlation properties and the diversity order. Either of the metrics can be used in practical design. And duration is not very sensitive to small diversity order mismatch between the actual channel and structure design

- Different metrics provide very similar patterns of resource structure and durations of each phase. This allows us to adopt one of the metrics, such as BER of repetition code, to design a practical resource structure.

For the systems with null-guard tones we obtain similar result and the same features as we conclude above.

For systems with large dimensionality (large $n_{\mathrm{B}}$ and $W$ ), the above characteristics provide useful guidelines for the resource structure design. First, it is an evolving structure with a maximum of three phases. Second, the band-type in the first phase and the interleaved-type in the third phase will maintain the same trend for any system dimensionality. However, the durations of the three phases and the mixed-type structures in the transition phase may differ as the system dimensionality varies (they are also affected by the channel correlation properties and diversity order) and they are the missing parts in the resource structure design for systems with large dimensionality. In the following sub-sections, we first verify the superiority of the evolving resource structure, and then develop the missing design aspects theoretically under a simplified system model.

\section{BER Performance of Different Resource Structures in a Simplified System Model}

The approach in Section III-B can only be used in small dimensionality due to its computational complexity. Fortunately, the result of optimal structure designs suggests us a simplified model. From Fig. 2, the selected SPRBs in three phases can be simply classified into two categories: remaining in the same band-type subchannel or becoming independent from the original band-type subchannel. And for those remaining in the same subchannel, we can assume they have the same

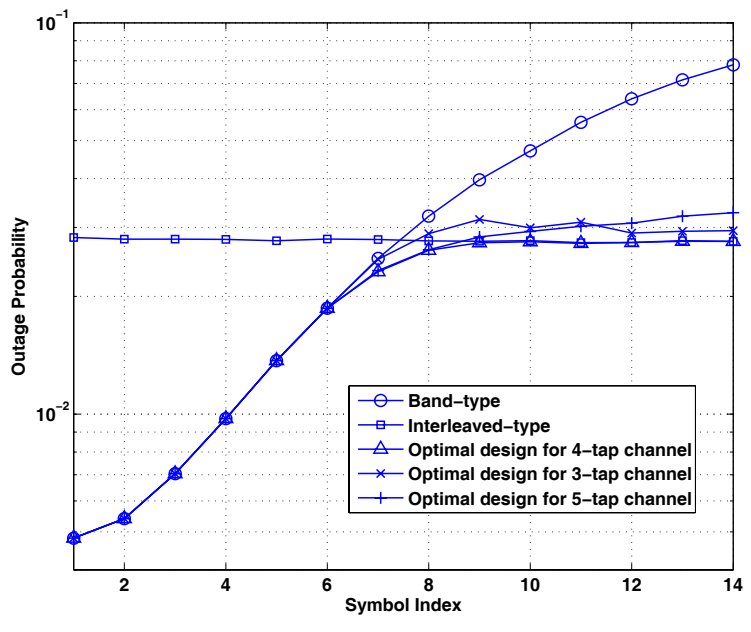

Fig. 4. Outage probability comparison in a 4-tap channel with $\mathrm{SNR}=3 \mathrm{~dB}$, $T_{\mathrm{s}}=71.36 \mu \mathrm{s}$ and $R_{T}=2$

channel power gain for simplicity. This simplified model can be applied to design the structure in not only small dimensionality cases but also large dimensionality cases.

In order to validate the advantage of the evolving resource structure without any complication, we compare three resource structures over the $n$th OFDM symbol. The first structure is the interleaved-type with $W$ SPRBs, which are spread out across the transmission band such that their channel gains can be considered as mutually independent, hence providing frequency diversity. The second structure is the band type which exploits multiuser diversity of order $R$ by allocating to the user its best subchannel based on the CSI knowledge. In this structure, all $W$ SPRBs of the subchannel are consecutively located such that their channel gains can be considered to be the same. The third structure is a mixture of the first two where $W_{\text {I }}$ SPRBs are spread out such that they have independent channel gains as in the first structure while the remaining $W_{\mathrm{B}}=W-W_{\mathrm{I}}$ SPRBs follow the same multiuser diversity exploitation as in the second structure.

As mentioned in Section III-B, BER of repetition code and outage probability give similar result in resource structure optimization. In order to illustrate the captured diversity, we use a repetition code with rate $1 / W$ to analyze the performance of the above three resource structures. For simplicity, we assume that each SPRB includes one subcarrier only and each structure carries one information bit per OFDM symbol with BPSK modulation. Let $\mathcal{I}$ denote the subcarrier index set of the resource structure under consideration. Then the effective channel power gain $G$ due to the repetition code is

$$
G=\sum_{i \in \mathcal{I}}|H[i, n]|^{2},
$$

and the BER for an average SNR is given by

$$
P_{e}=\int_{0}^{\infty} Q(\sqrt{2 g \cdot \mathrm{SNR}}) f_{G}(g) d g,
$$

where $Q(\cdot)$ is the Gaussian tail probability and $f_{G}(g)$ is the pdf of $G$. 


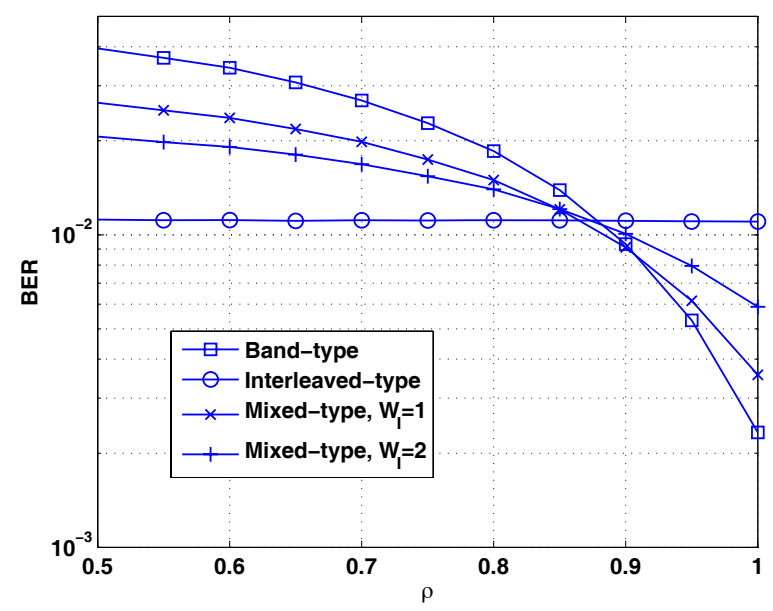

Fig. 5. Theoretical BER performance of different resource structures with diversity order of $4, W=4$ and SNR per subcarrier of $0 \mathrm{~dB}$.

1) Interleaved-type Structure: For the interleaved-type structure, as $\{H[i, n]: i \in \mathcal{I}\}$ are independent and identicallydistributed (i.i.d.) as $\mathcal{C N}\left(0,2 \sigma_{h}^{2}\right)$, the $G$ for the interleavedtype denoted by $G_{\mathrm{I}}$ has a Chi-square pdf with $2 W$ degrees of freedom as

$$
f_{G_{\mathrm{I}}}(g)=\frac{1}{\sigma_{h}^{2 W} 2^{W} \Gamma(W)} g^{W-1} e^{-g /\left(2 \sigma_{h}^{2}\right)},
$$

where $\Gamma(\cdot)$ is the Gamma function. Substituting (14) into (13) and solving as in [25] give BER for the interleaved-type as

$$
P_{e}=\left[\frac{1}{2}\left(1-\mu_{\chi}\right)\right]^{W} \sum_{k=0}^{W-1}\left(\begin{array}{c}
W-1+k \\
k
\end{array}\right)\left[\frac{1}{2}\left(1+\mu_{\chi}\right)\right]^{k},
$$

where

$$
\mu_{\chi}=\sqrt{\frac{2 \sigma_{h}^{2} \mathrm{SNR}}{1+2 \sigma_{h}^{2} \mathrm{SNR}}} .
$$

2) Band-type Structure: In the band-type structure, all $W$ subcarriers within a subchannel have the same channel gain, and the selected subchannel has a diversity order of $R$ due to multiuser diversity exploitation. The effective channel power gain of the selected subchannel based on the CSI knowledge is given by $\tilde{G} \triangleq \max \left\{W \cdot|H[i,-\mu]|^{2}: \forall i\right\}$. The pdf of $\tilde{G}$ can be obtained by the order statistics as

$$
f_{\tilde{G}}(b)=\frac{1}{W}\left[1-e^{-\frac{b}{2 \sigma_{h}^{2} W}}\right]^{R-1} \frac{R}{2 \sigma_{h}^{2}} e^{-\frac{b}{2 \sigma_{h}^{2} W}} .
$$

The corresponding channel power gain $G_{\mathrm{B}}$ (the subscript B denoting for the band-type) at the $n$th OFDM symbol evolves from $\tilde{G}$ according to (1) with $\rho_{i}[n+\mu]=\rho$. The conditional pdf of $G_{\mathrm{B}}$ given $\tilde{G}$ is a non-central Chi-square as

$$
f_{G_{\mathrm{B}} \mid \tilde{G}}(g \mid b)=\frac{1}{2 \eta W} e^{-\left(\rho^{2} b+g\right) /(2 \eta W)} I_{0}\left(\frac{\rho \sqrt{b g}}{\eta W}\right),
$$

where $\eta=\left(1-\rho^{2}\right) \sigma_{h}^{2}$, and $I_{0}(x)$ is the zeroth-order modified Bessel function of the first kind. Then we can evaluate the BER for the band-type as

$$
P_{e}=\int_{0}^{+\infty} \int_{0}^{+\infty} Q(\sqrt{2 g \cdot \mathrm{SNR}}) f_{G_{\mathrm{B}} \mid \tilde{G}}(g \mid b) f_{\tilde{G}}(b) d g d b .
$$

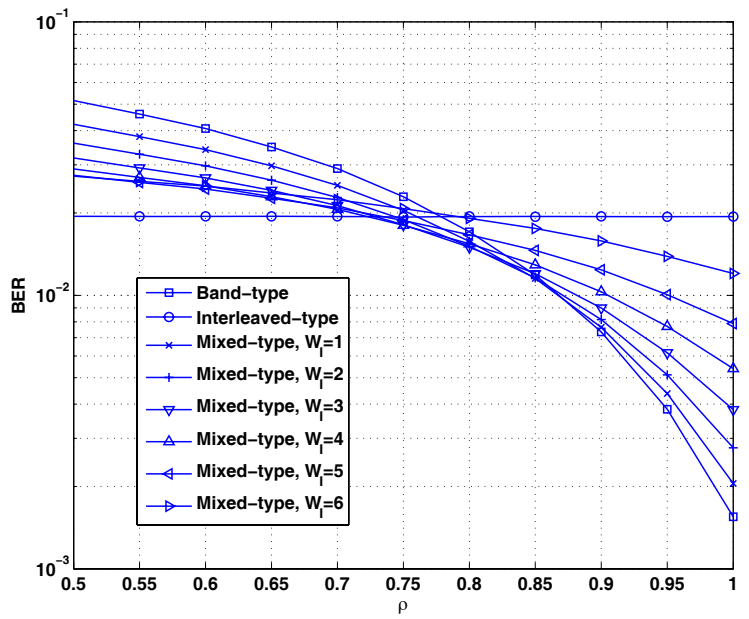

Fig. 6. Theoretical BER performance of different resource structures with diversity order of $8, W=8$ and SNR per subcarrier of $-5 \mathrm{~dB}$.

3) Mixed Structure: In the mixed structure, $W_{\text {I }}$ subcarriers have the same statistics as in the interleaved-type while the other $W_{\mathrm{B}}$ subcarriers follow the band-type, which is identical to the second phase of optimal designs in Section III-B. Denote the corresponding effective channel power gains due to $W_{\mathrm{I}}$ and $W_{\mathrm{B}}$ subcarriers by $G_{1}$ and $G_{2}$, respectively. Then $f_{G_{1}}(g)$, the pdf of $G_{1}$, is the same as $f_{G_{\mathrm{I}}}(g)$ except $W$ is replaced by $W_{\mathrm{I}}$, i.e., the degrees of freedom becomes $2 W_{\mathrm{I}}$ instead of $2 W$. Similarly, the pdf of $G_{2}$ is obtained as

$$
f_{G_{2}}(g)=\int_{0}^{\infty} \frac{W^{2}}{W_{\mathrm{B}}^{2}} f_{G_{\mathrm{B}} \mid \tilde{G}}\left(g W / W_{\mathrm{B}} \mid b\right) f_{\tilde{G}}\left(b W / W_{\mathrm{B}}\right) d b .
$$

The pdf of the effective channel power gain $G_{\mathrm{M}}=G_{1}+G_{2}$ of the mixed structure at the $n$th OFDM symbol is obtained by the convolution of $f_{G_{1}}(g)$ and $f_{G_{2}}(g)$. Substituting $f_{G_{\mathrm{M}}}(g)$ into (13) gives the BER expression for the mixed structure.

As examples, we set $W=4, R=4$ and $W=8, R=8$, both with $\sigma_{h}^{2}=1 / 2$. By numerical evaluation, we obtain the BERs of the structures as shown in Figs. 5 and 6, respectively at the SNR per subcarrier of $0 \mathrm{~dB}$ and $-5 \mathrm{~dB}$. If we pick up the structure with the best performance from all the candidates for different $\rho$, we will also obtain a three-phase evolving structure. When the correlation coefficient $\rho$ is high, the bandtype structure shows advantage over the other two structures. When $\rho$ is not so high, the mixed-type structure outperforms the other two structures. When $\rho$ is low, the interleaved-type structure has the best performance. Within the transmission frame, as time progresses, the channel correlation coefficient with respect to the CSI knowledge at the BS becomes smaller. The performance analysis shown in Figs. 5 and 6 clearly validates that to capture better diversity in multiuser timevarying frequency-selective fading channels, the subchannels should have an evolving resource structure. From the case of $W=8, R=8$, we can clearly see how the structure evolves in that the number of independent SPRBs increases step by step until interleaved-type appears. We also observe that the ranges of $\rho$ for the three phases in Fig. 5 with $W=4, R=4$ (under the simplified model) are very much similar to those in Fig. 2 (under the exact model). Thus, the simplified model 
provides a good approximation to the exact model and can be used to develop a practical resource structure design.

\section{Practical Resource Structure Design}

As obvious from the theoretical formulation in Section III-B, there is no theoretical closed-form solution for the user resource structure design. Numerical or simulation based solution to the theoretical formulation is feasible for OFDMA systems with small number of subcarriers but it is computationally prohibitive for large number of subcarriers. The simplified model which we proposed in Section III-C is a good approximation of the real system as pointed out in the previous section. Based on this simplified model, we will propose a new resource structure design approach (including a full version and a simplified version), which is suitable for both small and large dimensional systems. In this approach, the knowledge of diversity order, channel correlation properties, and pdf of the channel gains are required. Also, the proposed approach is robust to the small mismatch of these knowledge in practice.

The proposed practical design (evolving structure) is composed by the three structures as in Section III-C and consists of a maximum of three sequential phases as below:

- Multiuser diversity exploitation phase (a duration of $D_{M}$ OFDM symbols): the band-type structure is used in this phase to exploit multiuser diversity via CSI knowledge.

- Transition phase from multiuser diversity to frequency diversity (a duration of $D_{T}$ OFDM symbols): the mixed structure is used in this phase to exploit partial frequency diversity and partial multiuser diversity via tolerablyoutdated CSI knowledge.

- Frequency diversity exploitation phase (a duration of $D_{F}$ OFDM symbols): as the CSI knowledge becomes outdated, this phase just uses the interleaved structure to exploit frequency diversity.

In general, the duration of each phase depends on the mobile speed, carrier frequency, channel correlation characteristics, symbol duration, packet length, and CSI knowledge delay. Hence, at an extremely low mobile speed or static scenario, the evolving structure may have only one phase. In moderate or slow mobile speed case, the proposed structure may have two phases. The structure would have all three phases in a high mobile speed case. Especially, for the second phase, the mixed structure can include several stages (with different $W_{\mathrm{I}} / W_{\mathrm{B}}$ ) as shown in Fig. 6. Here, the design of the evolving resource structure can be divided into the following two strategies (both are necessary).

\section{A. Time-Domain Design}

First, let us describe the time-domain design which determines the time duration of each phase based on the channel temporal correlation and diversity order. It is a design to determine $\left\{\alpha_{i}\right\}$ which denotes the number of OFDM symbols in the $i$ th phase. We define $T_{\text {sym }}$ as the OFDM symbol duration including cyclic prefix (CP) and/or zero-padded guard interval. Let $\mathcal{T}_{A}$ denote the approximate duration of first phase and $\mathcal{T}_{B}$ denote the approximate duration of first two phases (delay of the channel information is included). The second phase may contain several (say $S$ ) stages, and $\mathcal{T}_{B, i}$ denotes the approximate duration from the time corresponding to CSI knowledge to the end of the $i$ th stage in the second phase. $\left[1, \rho_{\mathrm{A}}\right]$ and $\left(\rho_{\mathrm{A}}, \rho_{\mathrm{B}}\right]$, respectively, denote the ranges (in decreasing order) of the normalized channel temporal correlation coefficient of the first phase and second phase. The rest range belongs to the third phase. And in the second phase, $\rho_{\mathrm{B}, i}, i=1,2, \ldots, S$ defines the end of $i$ th stage in the second phase.

By the simplified model in Section III-C, we can easily obtain the BER curves, such as Fig. 6 with the parameter of diversity order. And we can read $\rho_{\mathrm{A}}, \rho_{\mathrm{B}}$ and $\rho_{\mathrm{B}, i}$ from the curves. Then we can find the corresponding $\mathcal{T}_{A}, \mathcal{T}_{B}$ and $\mathcal{T}_{B, i}$. If we use the Clark-Jakes' correlation model, we have

$$
\begin{aligned}
\mathcal{T}_{A} & =\frac{1}{2 \pi f_{m}} J_{0}^{-1}\left(\rho_{\mathrm{A}}\right), \\
\mathcal{T}_{B} & =\frac{1}{2 \pi f_{m}} J_{0}^{-1}\left(\rho_{\mathrm{B}}\right), \\
\mathcal{T}_{B, i} & =\frac{1}{2 \pi f_{m}} J_{0}^{-1}\left(\rho_{\mathrm{B}, i}\right) .
\end{aligned}
$$

Translating them into the numbers of OFDM symbols gives

$$
\begin{aligned}
N_{1} & \triangleq \operatorname{round}\left(\mathcal{T}_{A} / T_{\mathrm{sym}}\right), \\
N_{2} & \triangleq \operatorname{round}\left(\mathcal{T}_{B} / T_{\mathrm{sym}}\right), \\
N_{2, i} & \triangleq \operatorname{round}\left(\mathcal{T}_{B, i} / T_{\mathrm{sym}}\right) .
\end{aligned}
$$

Then we can use $N_{1}, N_{2}$, frame length $M$ and delay $\mu$ to describe the duration (in unit of the number of OFDM symbols) of each phase as follows:

$$
\begin{gathered}
\alpha_{1}=\min \left(M, N_{1}-\mu\right), \\
\alpha_{2}=\left\{\begin{array}{ll}
0, & \alpha_{1}=M \\
\min \left(N_{2}-N_{1}, M-\alpha_{1}\right), & \alpha_{1}<M
\end{array},\right. \\
\alpha_{3}=M-\alpha_{1}-\alpha_{2} .
\end{gathered}
$$

For the second phase, we can also obtain the number of symbols for each stage as

$$
\alpha_{2, i}=\left\{\begin{array}{ll}
N_{2,1}-N_{1}, & i=1 \\
N_{2, i}-N_{2, i-1}, & i=2, \ldots, S
\end{array} .\right.
$$

\section{B. Frequency-Domain Design}

This strategy focuses on subcarrier deployment of a subchannel in frequency-domain assuming that the channel coherence time is much smaller than the transmission frame length so that all three phases mentioned above exist. In the first phase, in order to capture multiuser diversity by taking advantage of CSI knowledge, the band-type structure is adopted. Since we assume that the BS exploits the CSI knowledge in its resource allocation, we can consider in the design that each user is allocated with its best subchannel. In the last phase, the CSI knowledge at the transmitter has become outdated, and we can treat it as no CSI at the transmitter. In this scenario, the interleaved-type structure is applied in order to exploit frequency diversity. Between the first phase and the last phase, the channel knowledge is partially outdated. However, it can still help us to obtain some multiuser diversity. Hence, we 
introduce a transition phase which adopts the mixed structure and its design is detailed in the following.

We adopt the simplified system model and the BER analysis from Section III-C to develop the resource structure design. Let $P_{e, n}\left(\rho[n+\mu] ; W_{I}[n], W_{B}[n]\right)$ represent the BER at the $n$th OFDM symbol for the structure with $W_{B}[n]$ SPRBs forming a band-type with multiuser diversity and $W_{I}[n]$ SPRBs forming an interleaved-type with frequency diversity, and $W=W_{\mathrm{I}}+W_{\mathrm{B}}$. Channel gains on all $W_{\mathrm{I}}[n]$ SPRBs are assumed to be independent mutually and also with the band-type SPRBs. For example, $P_{e, n}(\rho[n+\mu] ; W, 0)$ and $P_{e, n}(\rho[n+\mu] ; 0, W)$ correspond to the BER of the interleavedtype and band-type at the $n$th symbol, respectively. Then the resource structure design can be divided into two steps. In the first step, a right amount of mixture of the band and interleaved types is sought by the simplified model. In the second step, the SPRBs' locations are determined where not only the mixture amount obtained in the first step is maintained, but also the disjointness and the structure similarity (approximately the same or mirrored version) are enforced among all subchannels. For the first step, the optimal design under the simplified system model is given by

$$
\begin{aligned}
& \quad\left[W_{\mathrm{I}}[n], W_{\mathrm{B}}[n]\right]=\arg \min _{X, Y} P_{e, n}(\rho[n+\mu] ; X, Y), \\
& \text { s.t. } X+Y=W \text {, and } X, Y \in\{0,1, \ldots, W\} .
\end{aligned}
$$

For instance, Fig. 6 shows what should be the right mixture of the band and interleaved types at each stage of the first step based on the BER metric. Additionally, from the result in Section III-B, we have $\left[W_{I}[0], W_{B}[0]\right]=[0, W]$, and $J_{1}(0$, : )$=[0,1, \ldots, W-1], J_{k}(0,:)=(k-1) W+J_{1}(0,:)$.

Note that under this design, $W$ should not be larger than the frequency diversity order in order to closely resemble independent SPRBs of the interleaved-type. However, the case of $W$ larger than the diversity order can be handled by limiting the independent components in the above formulation and adjusting the signal power of these components to account for additional SPRBs in particular independent component(s).

For the second step, the SPRB indexes of the independent components should be spaced as far as possible among themselves as well as with the band-type ones. The above spacing measure is cyclical (of size $n_{\mathrm{B}}$ ) such that the spacing between $k$ and $k+m$ is $\min \left(m, n_{\mathrm{B}}-m\right)$. Based on the result from the first step, $J_{k}(n,:)$ can be divided into two subsets $J_{k}^{B}(n,:)$ and $J_{k}^{I}(n,:)$ of size $W_{B}[n]$ and $W_{I}[n]$, respectively, where $J_{k}^{B}(n,:) \in J_{k}(0,:)$ and $J_{k}^{I}(n,:) \notin J_{k}(0,:)$. For the $n$th symbol, divide each set $J_{k}(0,:)$ into two subsets $J_{k}^{B}$ and $J_{k}^{I}$ of size $W_{B}[n]$ and $W_{I}[n]$, respectively. Then, the design is given as

$$
\begin{aligned}
J_{k}^{B}(n,:) & =J_{k}^{B}, \forall k, n=1, \ldots, M-1, \\
J_{k}^{I}(n,:) & \in \cup_{m \neq k} J_{m}^{I}, \text { and } J_{k}^{I}(n,:) \cap J_{m}^{I}(n,:)=\emptyset, \\
& \forall k \neq m, n=1, \ldots, M-1,
\end{aligned}
$$

with the constraints that elements of $J_{k}^{I}(n,:)$ should be spaced as far as possible mutually and also from $J_{k}^{B}(n,:)$, and

$$
J_{k}(n,:)=\hat{J}_{1}(n,:)+D_{k}(n), \forall k .
$$

This second step design can easily be worked out manually or via a computer program.
For example, if $\left[W_{I}[0], W_{B}[0]\right]=[1,3]$, and $U=4$, $W=4, n_{\mathrm{B}}=16$, we may set $J_{1}(n,:)=[0,2,3,10]$, $J_{2}(n,:)=[4,6,7,14], J_{3}(n,:)=[1,8,9,11]$, and $J_{4}(n,:)=$ $[5,12,13,15]$, or $J_{1}(n,:)=[0,1,2,8], J_{2}(n,:)=[4,5,6,12]$, $J_{3}(n,:)=[3,9,10,11]$, and $J_{4}(n,:)=[7,13,14,15]$.

Due to the numerous combinatorial possibilities of the resource structures, it is impossible to provide a unique design solution for all scenarios. However, from the above design rules and guidelines, one can easily obtain a diversityenhanced resource structure.

\section{A Simplified Evolving Structure Design}

We have already proposed a practical approach to the evolving structure design in the previous section. However, due to multiple stages, this full version of the evolving structure is a bit complex. Here, we propose a simplified evolving structure which keeps the advantage of the evolving structure and enhances the flexibility in practical implementation.

Fig. 6 shows that performances of different mixed structures at the second phase are very similar. The simulation results (Figs. 7-10) in Section V also validate this. Additionally, there can be mismatches of the diversity order and the correlation coefficient between the actual values and those used in the design. These facts make the precise division of the second phase into several stages impractical and unnecessary. Hence, we simplify the second phase as one stage by adopting one of the mixed structures. Then the evolving structure is simplified into a three-stage structure, where the first and the last phases keep the same as the original evolving structure and the second phase is reduced to only one stage.

Thus, we only need to revise the design for the second phase. For the time domain design, we only need to obtain $\alpha_{2}$ which can be computed by the same approach as in Section IV-A. For the frequency domain design, we can pick up one of the candidate stages in the second phase of the original evolving structure as the simplified frequency domain design, e.g., the middle stage.

\section{An Example of Evolving Structure Design}

As an example, we consider the OFDMA system with $N=$ 256 subcarriers, which is the same as the DFT size. In the system, we assume $U=8$ and $W=8$. Each SPRB includes four subcarriers. We model the channel as eight uncorrelated Rayleigh fading taps, which have an exponential power delay profile with a $3 \mathrm{~dB}$ per tap decay factor. The frame length is $M=14$ symbols.

Using the simplified model, we can obtain the BER performance curves shown in Fig. 6. Let us do the time-domain design first. Then from the figure, we can easily obtain the number of stages and the correlation segmentation of each stage as shown in Table I. For the Jakes' model, by (21)(30) we obtain $\alpha$ for different speeds as in Table I. Here, we assume that the carrier frequency is $2 \mathrm{GHz}$ and the LTE symbol duration $\left(T_{\mathrm{sym}}=71.36 \mu \mathrm{s}\right)$ is adopted.

For the frequency-domain design, there are two steps. First, we can obtain $\left\{W_{\mathrm{I}}[n], W_{\mathrm{B}}[n]\right\}$ from Fig. 6 , which is given in Table I. Second, we should separate the SPRBs of the independent components as far as possible. We introduce a 


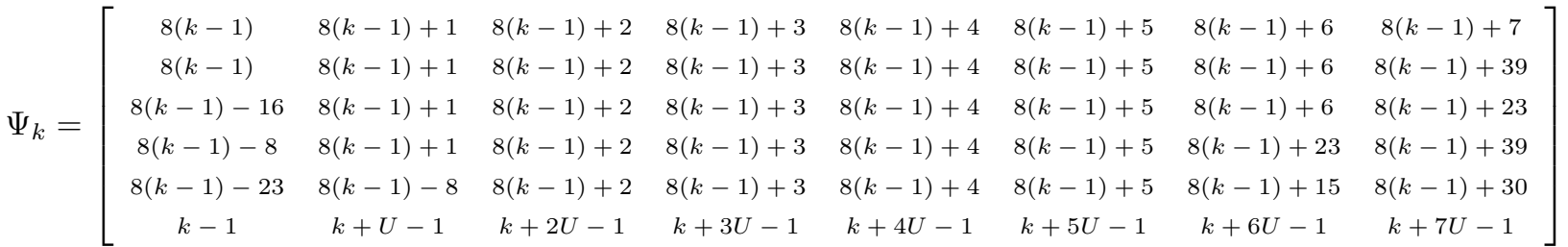

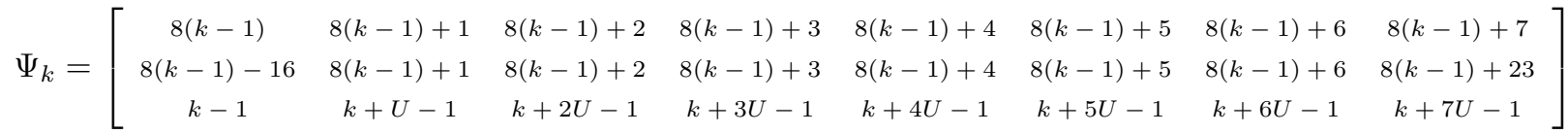

$D \times W$ matrix $\tilde{\boldsymbol{J}}_{k}$ which denotes the template SPRB index set for the $k$ th E-PRB or subchannel where $k=1,2, \ldots, U$. The $d$ th row of $\tilde{\boldsymbol{J}}_{k}$ is $J_{k, d}$ denoting the SPRB index set of the $d$ th stage of the $k$ th E-PRB index template. According to the result of time-domain design, this structure includes six stages (four stages in second phase). In order to space the $W_{\mathrm{I}}[n]$ SPRBs mutually as far as possible, we use the index matrix (35) to allocate the SPRBs as

$$
\tilde{\boldsymbol{J}}_{k}=\Psi_{k} \bmod 64,
$$

where $k=1,2, \ldots, U$, and $\Psi_{k}$ is given by (35)

We can also have the simplified evolving structure for this system by revising the original evolving structure as follows. For the time domain design, we can have $\alpha_{2}$ from Table I as $\alpha_{2}=\sum_{i} \alpha_{2, i}$. For the frequency domain design of the simplified structure, we only need to change (35) into (36).

\section{Simulation Results And Discussion}

\section{A. Simulation Setting}

We use the parameters of the LTE system to validate the advantages of the proposed evolving structures over the existing ones. Here, we consider the OFDMA system with $N=256$ subcarriers, which is the same as the DFT size. The simulation parameters we used are adopted from the LTE system: carrier frequency is $2 \mathrm{GHz}$, subcarrier spacing is 15 $\mathrm{kHz}$, and the symbol duration including the $\mathrm{CP}$ is $71.36 \mu \mathrm{s}$. The frame (packet) length is $M=14$ OFDM symbols.

The channel model is a frequency-selective time-varying channel. There are eight uncorrelated Rayleigh fading taps, which have an exponential power delay profile with a 3 $\mathrm{dB}$ per tap decay factor. The channel gains are assumed to be static within one OFDM symbol duration but vary from symbol to symbol according to the Clark-Jakes' Doppler spectrum. In order to evaluate the performance in various mobile environments, we use seven mobile speeds ranging from $3 \mathrm{~km} / \mathrm{h}$ to $350 \mathrm{~km} / \mathrm{h}$. The parameters related to the time domain design are shown in Table I.

The industrial standard convolutional code with rate $1 / 2$, constraint length 7 , and generator matrix $[133,177]$ is applied across the subcarriers. The bit interleaver described in Appendix $\mathrm{A}$ is applied with $d=56$. And both quadrature phaseshift keying (QPSK) and 16 quadrature amplitude modulation (16-QAM) are used. The Viterbi decoder [26] is adopted in the receiver. The parameters of the evolving structure are $U=8$ subchannels (PRBs), $n_{\mathrm{B}}=64$ SPRBs, $W=n_{\mathrm{B}} / U=8$ SPRBs per PRBs, and 4 subcarriers per SPRBs. Since the number of independent channel taps and the number of subchannels are both 8 , the diversity order $R$ is 8 in this system (we take the minimum of the number of independent channel taps and the number of subchannels as the value of diversity order in the design). As performance references, we also evaluate the existing resource structures - the bandtype and the interleaved-type. For the evolving structure's frequency domain design, (35) and (36) are respectively used for the original and simplified evolving structures.

\section{B. Simulation Results}

The plots of BER versus mobile speed is presented in Figs. 7 and 9, respectively for QPSK at the information bit energy to noise spectral density ratio $\left(E_{b} / N_{0}\right)$ of $5 \mathrm{~dB}$ and 16-QAM at $E_{b} / N_{0}$ of $9 \mathrm{~dB}$. And the plots of BER versus $E_{b} / N_{0}$ are shown in Figs. 8 and 10. At a very low mobile speed, the proposed structures (original and simplified evolving structures) have the same performance as the bandtype while the interleaved-type has a performance loss. When the speed becomes very high, the performance of the bandtype is significantly affected, while the interleaved-type enjoys a good performance. And the proposed structures have even a better performance than the interleaved-type in this case, due to multiuser diversity exploitation at the beginning of the frame. At medium mobile speeds, the proposed structures outperform both conventional structures due to its better diversity exploitation. Further, the simplified evolving structure gives almost the same performance as the original evolving structure at all evaluated cases, which validates the simplified evolving structure design. Therefore, we can apply the simplified evolving structure in practical systems instead of the original one so that the complexity can be reduced. Additionally, we observe that BERs of interleaved-type and evolving-type (at high mobile speed) decrease as mobile speed increases due to larger time diversity at a higher mobile speed.

In order to test the sensitivity to the speed mismatch of the proposed approach, we evaluate the designs for $160 \mathrm{~km} / \mathrm{h}$, $200 \mathrm{~km} / \mathrm{h}$ and $240 \mathrm{~km} / \mathrm{h}$ respectively in the channel with the actual speed of $200 \mathrm{~km} / \mathrm{h}$ and QPSK. The results are shown in Fig. 11. The results of the three designs are very similar, which illustrates that the proposed approach is not very sensitive to the speed mismatch. Additionally, the design 
TABLE I

TIME-DOMAIN DESIGN FOR LTE SYSTEM

\begin{tabular}{||c|c|c|c|c|c|c|c||}
\hline Phase and Stage & $\rho$ & $W_{\mathrm{B}} / W_{\mathrm{I}}$ & $\alpha(50 \mathrm{~km} / \mathrm{h})$ & $\alpha(80 \mathrm{~km} / \mathrm{h})$ & $\alpha(120 \mathrm{~km} / \mathrm{h})$ & $\alpha(200 \mathrm{~km} / \mathrm{h})$ & $\alpha(350 \mathrm{~km} / \mathrm{h})$ \\
\hline Phase 1 & 0.871 & $8 / 0$ & $\alpha_{1}=14$ & $\alpha_{1}=10$ & $\alpha_{1}=7$ & $\alpha_{1}=4$ & $\alpha_{1}=2$ \\
\hline P 2, S 1 & 0.846 & $7 / 1$ & $\alpha_{2,1}=0$ & $\alpha_{2,1}=2$ & $\alpha_{2,1}=1$ & $\alpha_{2,1}=0$ & $\alpha_{2,1}=0$ \\
\hline P 2, S 2 & 0.809 & $6 / 2$ & $\alpha_{2,2}=0$ & $\alpha_{2,2}=1$ & $\alpha_{2,2}=0$ & $\alpha_{2,2}=1$ & $\alpha_{2,2}=1$ \\
\hline P 2, S 3 & 0.756 & $5 / 3$ & $\alpha_{2,3}=0$ & $\alpha_{2,3}=1$ & $\alpha_{2,3}=2$ & $\alpha_{2,3}=1$ & $\alpha_{2,3}=0$ \\
\hline P 2, S 4 & 0.723 & $4 / 4$ & $\alpha_{2,4}=0$ & $\alpha_{2,4}=0$ & $\alpha_{2,4}=0$ & $\alpha_{2,4}=0$ & $\alpha_{2,4}=0$ \\
\hline Phase 3 & 0 & $0 / 8$ & $\alpha_{3}=0$ & $\alpha_{3}=0$ & $\alpha_{3}=4$ & $\alpha_{3}=8$ & $\alpha_{3}=11$ \\
\hline
\end{tabular}

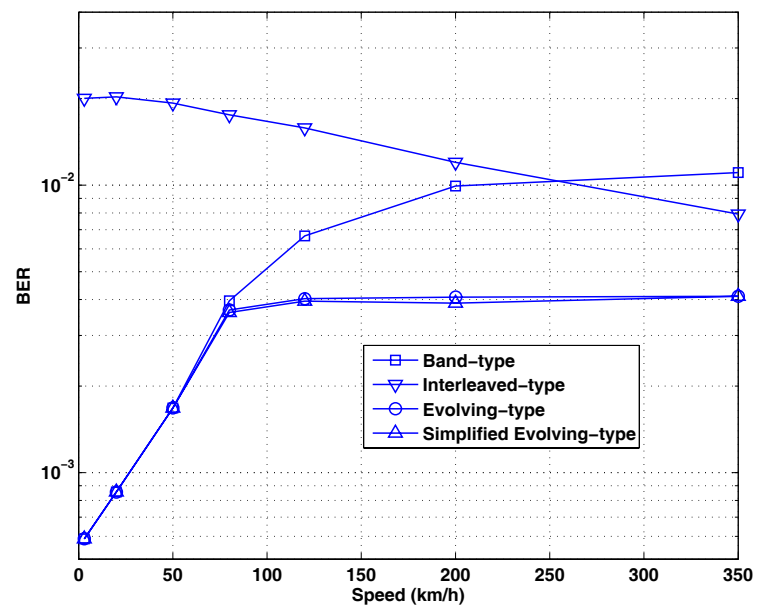

Fig. 7. BER performance comparison under various mobile speeds between the proposed evolving resource structure and the existing resource structures with $E_{b} / N_{0}=5 \mathrm{~dB}, M=14$, QPSK and rate $1 / 2$ convolutional code.

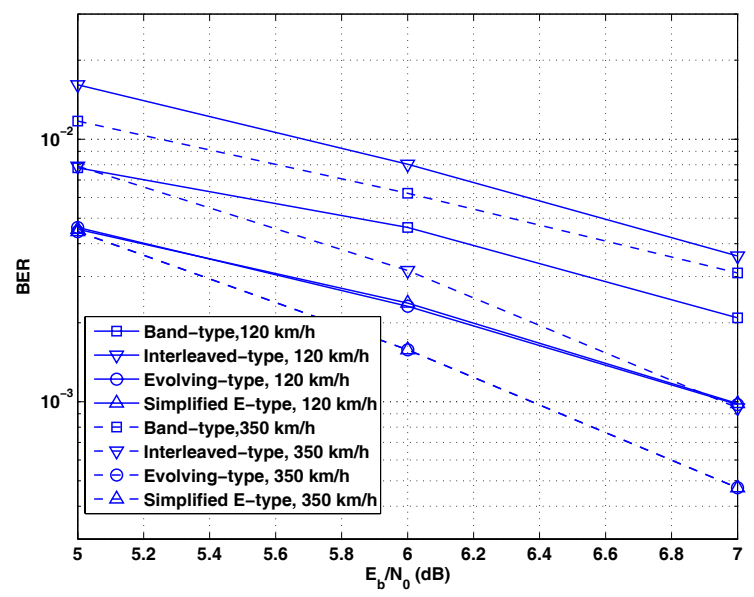

Fig. 8. BER performance comparison under various SNRs between the proposed evolving resource structure and the existing resource structures with $M=14$, QPSK and rate $1 / 2$ convolutional code.

for $160 \mathrm{~km} / \mathrm{h}$ evolves slower than the design for $200 \mathrm{~km} / \mathrm{h}$, while the design for $240 \mathrm{~km} / \mathrm{h}$ evolves faster. The design for $200 \mathrm{~km} / \mathrm{h}$ outperforms the other two a bit in the channel with actual speed of $200 \mathrm{~km} / \mathrm{h}$, which illustrates that the design for $200 \mathrm{~km} / \mathrm{h}$ is a near-optimal design for the channel with actual speed of $200 \mathrm{~km} / \mathrm{h}$. Thus, the proposed approach is validated as a near-optimal approach.

\section{CONCLUSiOnS}

We have proposed a new approach of enhancing diversities by means of user resource structure (or user channeliza-

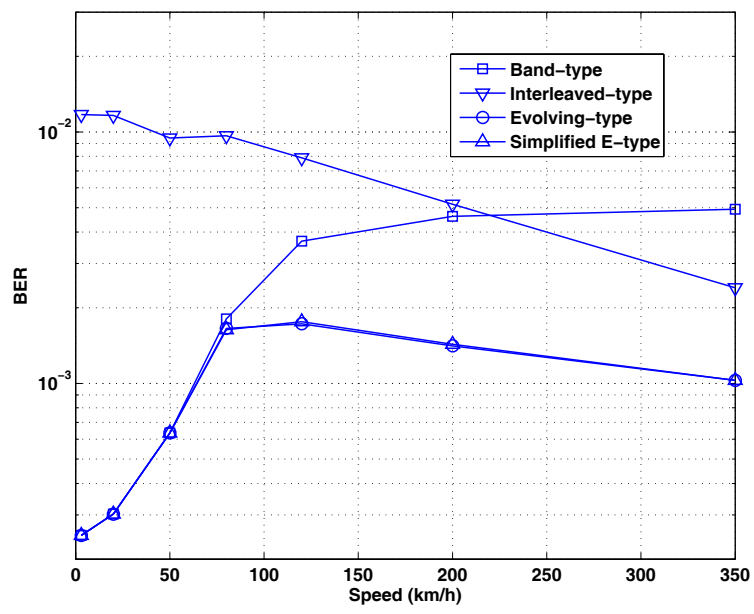

Fig. 9. BER performance comparison under various mobile speeds between the proposed evolving resource structure and the existing resource structures with $E_{b} / N_{0}=9 \mathrm{~dB}, M=14,16-\mathrm{QAM}$ and rate $1 / 2$ convolutional code.

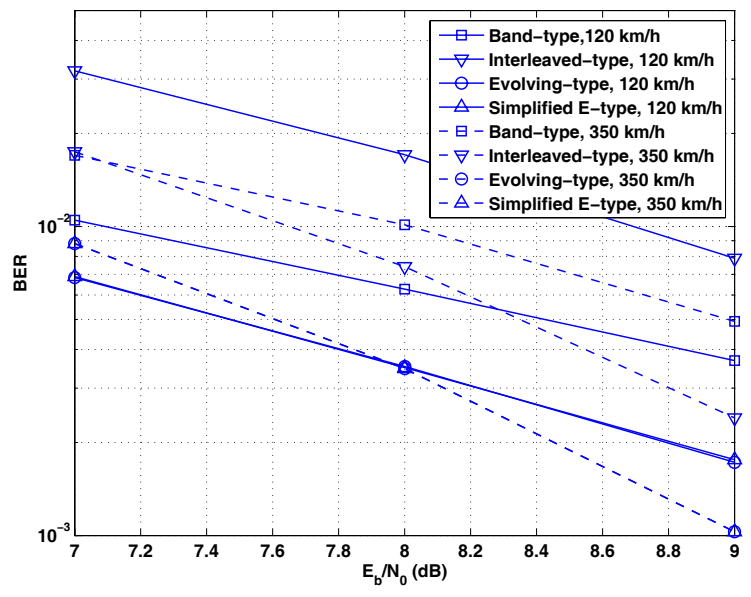

Fig. 10. BER performance comparison under various SNRs between the proposed evolving resource structure and the existing resource structures with $M=14,16-\mathrm{QAM}$ and rate $1 / 2$ convolutional code.

tion) design for OFDMA systems in multiuser time-varying frequency-selective fading channels. We developed theoretical formulations of the user resource structure design using BER and outage probability as design metrics, and obtained optimal resource structures for small-scale systems numerically. This study revealed several important characteristics of optimal resource structures and provided guidelines for practical resource structure designs. We further developed a simplified system model to handle resource structure designs for largescale systems, and observed from the performance results that the simplified model yielded close-to-optimal performance. In 


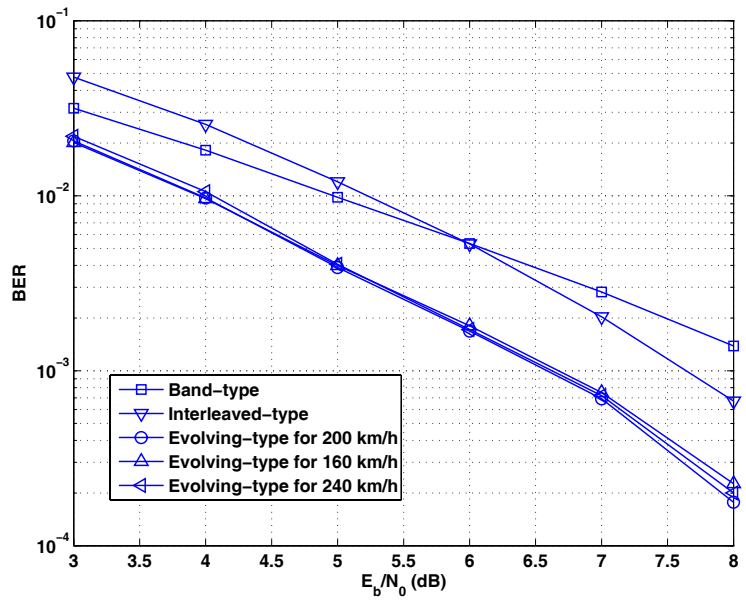

Fig. 11. BER performance under mobile speed mismatch in the channel with $200 \mathrm{~km} / \mathrm{h}, M=14$, QPSK and rate $1 / 2$ convolutional code.

addition, a simplified resource structure design was devised which maintained almost the same performance as the full version of the resource structure design while enjoying simpler implementation complexity and flexibility. The simulation results also illustrated that the proposed designs were not sensitive to the mismatches in diversity order and mobile speeds. The proposed resource structure may contain up to three phases of diversity exploitation (multiuser diversity, partial multiuser and frequency diversities, and frequency diversity) depending on the mobile environment under consideration. Thus, the user resource structure with enhanced diversity starts with a band-type and may evolve into a mixed type and then an interleaved-type at a later part of the transmission frame if the channel varies substantially within the frame. The simulation results under the LTE system setting validated that the proposed evolving resource structure provided significant BER performance improvement over the existing resource structures. In brief, the proposed approach opens up a viable additional dimension for diversity enhancement approaches.

\section{APPENDIX A \\ INTERLEAVER DESIGN}

In order to illustrate the advantage of the evolving structure, we adopt an interleaver including two permutations revised from the interleavers used in WiMAX [4] and IEEE 802.11a [27]. The index of the coded bit before the first permutation shall be denoted by $k$, and $i$ is the index after the first and before the second permutation; and $j$ is the index after the second permutation, just prior to modulation mapping. It should be noted that the index here is ordered within one frame. The first permutation is defined by the rule

$$
i=\left(N_{\mathrm{BPF}} / d\right)(k \bmod d)+\lfloor k / d\rfloor, k=0,1, \ldots N_{\mathrm{BPF}}-1 .
$$

The function $\lfloor$.$\rfloor denotes the largest integer not exceeding the$ argument. And $N_{\mathrm{BPF}}$ is the number of bits per frame. The second permutation is defined by the rule

$$
\begin{aligned}
& j=s \times\lfloor i / s\rfloor+\left(i+N_{\mathrm{BPF}}-\left\lfloor d \times i / N_{\mathrm{BPF}}\right\rfloor\right) \bmod s, \\
& i=0,1, \ldots, N_{\mathrm{BPF}}-1 .
\end{aligned}
$$

The values of $s$ are determined by the number of coded bits per subcarrier, $N_{\mathrm{BPSC}}$, according to

$$
s=\max \left(N_{\mathrm{BPSC}} / 2,1\right) .
$$

The deinterleaver is also defined by two permutations. Here, the index of the original received bit before the first permutation shall be denoted by $j ; i$ shall be the index after the first and before the second permutation; and $k$ shall be the index after the second permutation, just prior to delivering the coded bit to convolutional (Viterbi) decoder. The first permutation is defined by the rule

$$
\begin{array}{r}
i=s \times\lfloor j / s\rfloor+\left(j+\left\lfloor d \times j / N_{\mathrm{BPF}}\right\rfloor\right) \bmod s, \\
j=0,1, \ldots, N_{\mathrm{BPF}}-1,
\end{array}
$$

where $s$ is defined in (39). The second permutation is defined by the rule

$$
\begin{array}{r}
k=d \times i-\left(N_{\mathrm{BPF}}-1\right)\left\lfloor d \times i / N_{\mathrm{BPF}}\right\rfloor, \\
i=0,1, \ldots, N_{\mathrm{BPF}}-1 .
\end{array}
$$

\section{REFERENCES}

[1] WP5D, "Draft report on requirements to technical performance for IMT-Advanced radio interface(s) [IMT.TECH]," Working Party 5D SubWorking Group Radio Aspects, July 2008.

[2] 3GPP, Long-Term Evolution (LTE), "Physical Channels and Modulation," (Relase 8), 3GPP TS 36.211 v1.2.0, June 2007.

[3] 3GPP, LTE-Advanced, "Requirements for further advancements for EUTRA," (Release 8), 3GPP TR 36.913 v8.0.1, Mar. 2009.

[4] IEEE 802.16e, "Air Interface for Fixed and Mobile Broadband Wireless Access Systems."

[5] D. Huang, K. B. Letaief, and J. Lu, "Bit-interleaved time-frequency coded modulation for OFDM systems over time-varying channels," IEEE Trans. Commun., vol. 53, no. 7, pp. 1191-1199, July 2005.

[6] C. R. N. Athaudage, J. Wang, and A. D. S. Jayalath, "An efficient framework to exploit frequency diversity in OFDM: precoding with adaptive subcarrier selection," in Proc. IEEE PIMRC, Sep. 2006, pp. $1-5$.

[7] M. L. McCloud, "Optimal binary spreading for block OFDM on multipath fading channels," in Proc. IEEE WCNC, Mar. 2004, pp. 965970.

[8] B. Bai, W. Chen, Z. Cao, and K. B. Letaief, "Achieving high frequency diversity with subcarrier allocation in OFDMA systems," in Proc. IEEE GLOBECOM, Nov. 2008, pp.1-5.

[9] C. Y. Wong and R. S. Cheng, "Multiuser OFDM with adaptive subcarrier, bit, and power allocation," IEEE J. Sel. Areas Commun., vol. 17, no. 10 , pp. $1747-1758$, Oct. 1999.

[10] R. Knopp and P. Humblet, "Information capacity and power control in single-cell multiuser communications," in Proc. IEEE ICC, June 1995, pp. 331-335.

[11] D. N. C. Tse, "Optimal power allocation over parallel Gaussian broadcast channels," in Proc. IEEE ISIT, June 1997, p. 27.

[12] K. D. Lee and V. Leung, "Fair allocation of subcarrier and power in an OFDMA wireless mesh network," IEEE J. Sel. Areas Commun., vol. 24, no. 11, pp. 2051-2060, Nov. 2006.

[13] G. Li and H. Liu, "Downlink radio resource allocation for multi-cell OFDMA system," IEEE Trans. Wireless Commun., vol. 5, no. 12, pp. 3451-3459, Dec. 2006

[14] S. J. Lee, "Trade-off between frequency diversity gain and frequency selective scheduling gain in OFDMA systems with spatial diversity," IEEE Commun. Lett., vol. 11, no. 6, pp. 507-509, June 2007.

[15] R. Chemaly, K. Letaief, and D. Zeghlache, "Adaptive resource allocation for multiuser MIMO/OFDM networks based on partial channel state information," in Proc. IEEE GLOBECOM, Nov. 2005, pp. 1662-1672.

[16] X. Qin and R. Berry, "Distributed approaches for exploiting multiuser diversity in wirelessnetworks," IEEE Trans. Inf. Theory, vol. 52, no. 2, pp. 392-413, Feb. 2006.

[17] D. Wang, H. Minn, and N. Al-Dhahir, "A distributed opportunistic access scheme and its application to OFDMA systems," IEEE Trans. Commun., pp. 738-746, Mar. 2009. 
[18] G. Jongren and M. Skoglund, "Quantized feedback information in orthogonal space-time blockcoding," IEEE Trans. Inf. Theory, vol. 50, no. 10, pp. 2473-2486, Oct. 2004.

[19] V. Lau, Y. Liu, and T.-A. Chen, "On the design of MIMO block fading channels with feedback-link capacity constraint," IEEE Trans. Inf. Theory, vol. 50, no. 10, pp. 2473-2486, Jan. 2004.

[20] J. Choi and R. W. Heath, "Interpolation based transmit beamforming for MIMO-OFDM with limited feedback," IEEE Trans. Signal Process., vol. 53, no. 11, pp. 4125-4135, Nov. 2005.

[21] T. Pande, D. J. Love, and J. V. Krogmeier, "Reduced feedback MIMOOFDM precoding and antenna selection," IEEE Trans. Signal Process., vol. 55, no. 5, pp. 2284-2293, May 2007.

[22] D. Goeckel, "Adaptive coding for time-varying channels using outdated fading estimates," IEEE Trans. Commun., vol. 47, no. 6, pp. 844-855, June 1999.

[23] F.-S. Chu and K.-C. Chen, "Fair adaptive radio resource allocation of mobile OFDMA," in Proc. IEEE PIMRC, Sep. 2006. pp. 1-5.

[24] D. Piazza and L. B. Milstein, "Analysis of multiuser diversity in timevarying channels," IEEE Trans. Wireless Commun., vol. 6, no. 12, pp. 4412-4419, Dec. 2007.

[25] J. G. Proakis and M. Salehi, Digital Communication, 5th edition. McGraw-Hill, 2008, pp. 851-859.

[26] T. May, H. Rohling, and V. Engels, "Performance analysis of Viterbi decoding for 64-DAPSK and 64-QAM modulated OFDM signals," IEEE Trans. Commun., vol. 46, no. 2, pp. 182-190, Feb. 1998.

[27] IEEE 802.11, "Wireless LAN Medium Access Control and Physical Layer Specifications."

[28] H. Minn and C.-C. Chong, "User resource structure design with enhanced diversity for OFDMA in time-varying channels," in Proc. IEEE WCNC, Apr. 2009, pp. 646-651.

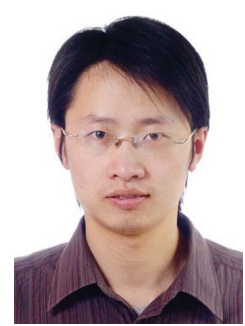

Wenxun Qiu (S'09) received the B.E. and M.S degrees in electrical engineering from the Harbin Institute of Technology in 2005 and 2008, respectively. He is currently working towards his Ph.D. degree in the electrical engineering department at the University of Texas at Dallas. His research interests include resource allocation in multicarrier systems, cognitive radio networks, and duplexing schemes design.

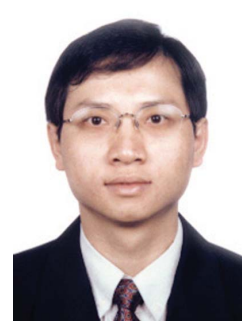

Hlaing Minn (S'99-M'01-SM'07) received the B.E. degree in electronics from the Yangon institute of Technology, Yangon, Myanmar, in 1995, the M.Eng. degree in telecommunications from the Asian Institute of Technology (AIT), Pathumthani, Thailand, in 1997, and the Ph.D. degree in electrical engineering from the University of Victoria, Victoria, BC, Canada, in 2001. He was a post-doctoral fellow at the University of Victoria, Jan.-Aug. 2002. He has been with the Erik Jonsson School of Engineering and Computer Science, the University of Texas at Dallas, Richardson, since Sept. 2002, and currently is an Associate Professor. His research interests include wireless communications, statistical signal processing, error control, detection, estimation, synchronization, signal design, cross-layer design, cognitive radios, and wireless health-care applications. Prof. Minn is an Editor for the IEEE TRANSACTIONS ON COMMUNICATIONS and the International Journal of Communications and Networks. He served as a Co-Chair of the Wireless Access Track for IEEE VTC 2009 (Fall).

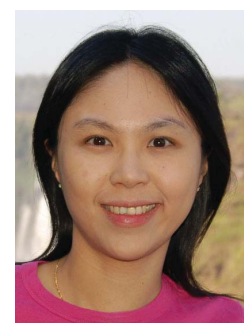

Chia-Chin Chong (S'00-M'03-SM'08) is a Senior Manager at Orange France Telecom in San Francisco, CA. Prior to joining Orange, she spent over five years at NTT DOCOMO USA Labs and two years at Samsung Electronics R\&D. Dr. Chong has done research in cellular systems, short range communications, wireless home energy management, and wireless healthcare. She has been an invited speaker and panelist in many international conferences. Dr. Chong has authored a book chapter and published more than 90 international journals, conference papers, and international standard contributions. She also has more than 30 granted and pending patents.

Dr. Chong has received numerous awards including the IEE Prize and the IEE Vodafone Research Award in 1999 and 2001, respectively, the IEEE International Conference on Ultra-Wideband (ICUWB) Best Paper Award and the DOCOMO USA Labs President Award in 2006, and the URSI Young Scientist Award and the DOCOMO USA Labs President Award in 2008. Dr. Chong was honored with The Outstanding Young Malaysian Award in 2006 (the most prestigious achievement award for people under the age of 40 in Malaysia) by the Junior Chamber International and Ministry of Youth and Sports, Malaysia, under the category "Scientific and Technological Development."

Currently, Dr. Chong serves as an Editor for the IEEE TRAnSACTIONS on Wireless COMMUNICATIONS, and Associate Editor for the IEEE TRANSACTIONS ON VeHICULAR TECHNOLOGY and the EURASIP Journal on Wireless Communications and Networking (JWCN). She has served as a committee member and organizer of various international conferences including TPC Co-Chair for the Broadband Wireless Access Symposium of IEEE ICCCN 2007, TPC Co-Chair for the Wireless Communications Symposium of IEEE ICC 2008, Publicity Co-Chair for IEEE PIMRC 2008, Sponsorship Chair for CrownCom 2008, and Tutorial Chair for IEEE ICCCN 2009. She was also Former Chair for the DG-EVAL Channel Model standardization group within the ITU-R WP5D for IMT systems. She is a Senior Member of the IEEE. 